\title{
ON COMMON FIXED AND PERIODIC POINTS OF COMMUTING FUNCTIONS
}

\author{
ALIASGHAR ALIKHANI-KOOPAEI \\ Mathematics Department \\ University of Isfahan \\ Isfahan, Iran
}

(Received March 8, 1996 and in revised form March 30, 1996)

\begin{abstract}
It is known that two commuting continuous functions on an interval need not have a common fixed point. It is not known if such two functions have a common periodic point. In this paper we first give some results in this direction. We then define a new contractive condition, under which two continuous functions must have a unique common fixed point.
\end{abstract}

KEY WORDS AND PHRASES: FIXED POINTS, PERIODIC POINTS, COMMUTING FUNCTIONS , MINIMAL SETS.

1992 AMS SUBJECT CLASSIFICATION CODES: 26A16, 47H10.

\section{INTRODUCTION.}

For some time there was a rather well known conjecture that if $f$ and $g$ are continuous commuting selfmaps of $[0,1]$ (i.e. $f(g(x))=g(f(x))$ ), then they have a common fixed point. W.M. Boyce [1] and J.P. Huneke [2] answered this conjecture in the negative by constructing a pair of commuting continuous functions which have no common fixed point. It is easy to see that their pair of commuting continuous functions have a common periodic point. In fact for the Boyce's example zero is a common periodic point. Thus one may conjecture the following:

CONJECTURE 1.1. If $f$ and $g$ are commuting continuous selfmaps of $[0,1]$, then they must have a common periodic point.

Even though we believe that the answer to the above conjecture is also negative, at present we are not able to construct a counterexample.

A.J. Schwartz [3] proved that if $f$ and $g$ are continuous functions of $[0,1]$ into itself that 
commute, then there always exists a point $x$ such that $x=f(x)=g^{n}(x)$ for some positive integer $n$, under the additional assumption that $f$ has a continuous derivative. In this paper we first give some results in this direction. Then we define a property $P_{1}$ and show that under this condition, two commuting continuous functions must have a unique common fixed point.

We begin with some preliminaries. The orbit of $x$ under $g$ (i.e. the set $\left\{g^{k}(x): k \geq 0\right\}$ ) and its closure are denoted by $O(g, x)$ and $\overline{O(g, x)}$, respectively. The set of cluster points of $O(g, x)$ is denoted by $\omega(g, x)$. A subset $Y$ of $I$ is called invariant under $g$ if $g(Y) \subseteq Y$. A closed, invariant, nonempty subset of $I$ is called minimal if it contains no proper subset that is also closed, invariant and nonempty. A point $x$ is called a recurrent point of $g$ if $x$ belongs to $\omega(g, x)$. Throughout $f^{n}$ denotes the $n$ fold composition of $f$ with itself. The sets $P(f), R(f)$ and $F(f)$ are the sets of periodic points of $f$, recurrent points of $f$, and the fixed points of $f$, respectively. We state some known facts concerning minimal sets. Every closed, invariant, nonempty subset of $I$ contains a minimal set. If $Y$ is a minimal set, then $Y \subseteq R(f)$. If $Y$ is a minimal set, which is not the orbit of a periodic point, then $Y$ is perfect. A minimal set is nowhere dense.

\section{THE MAIN RESULTS.}

THEOREM 2.1. Let $f$ and $g$ be two commuting continuous selfmaps of the unit interval. If $f$ and $g$ have no common periodic points, then for any two positive integers $m$ and $n$ the set $A_{m, n}=\left\{x: f^{n}(x)=g^{m}(x)\right\}$ is uncountable.

PROOF. First we show that $A_{m, n}$ is not empty. If for some $m$ and $n, A_{m, n}$ is empty, the continuity of $f$ and $g$ permits us to assume without loss of generality, that

$$
f^{n}(x)<g^{m}(x)
$$

for all $x \in I$. Since $g^{m}(1) \leq 1$, the set $S=\left\{x \in I: g^{m}(x) \leq x\right\}$ is not empty. Thus, since $S$ is closed, it has a minimum element $c$. Clearly, $c=g^{m}(c)$. Hence $f^{n}(c)=f^{n}\left(g^{m}(c)\right)=g^{m}\left(f^{n}(c)\right)$, so that $f^{n}(c) \in S$. Consequently $f^{n}(c) \geq c=g^{m}(c)$. Since $f^{n}(c) \geq g^{m}(c)$ contradicts (2.1), the assertion that $A_{m, n}$ is empty is false. Now suppose that $x \in A_{m, n}$. Then $f^{n}(x)=g^{m}(x)$ hence, $f^{n}(f(x))=f\left(g^{m}(x)\right)=g^{m}(f(x))$ and $f^{n}(g(x))=g\left(f^{n}(x)\right)=g\left(g^{m}(x)\right)=g^{m}(g(x))$. Thus if $x \in A_{m, n},\{f(x), g(x)\} \subseteq A_{m, n}$ and $f^{p n}(x)=g^{p m}(x)$ for each positive integer $p$. From this it follows that $O(g, x)$ is contained in $A_{m, n}$ whenever $x \in A_{m, n}$. The set $\overline{O(g, x)}$ is an invariant set under $g$. Suppose $A_{1}$ is a minimal set contained in $\overline{O(g, x)}$. Since $f$ and $g$ have no common periodic points, the set $A_{1}$ cannot be the orbit of a periodic point. Hence $A_{1}$ is a perfect set contained in $A_{m, n}$, implying that $A_{m, n}$ is uncountable.

COROLLARY 2.1. Let $f$ and $g$ be two commuting continuous selfmaps of the unit interval. If for some positive integers $m$ and $n$, the set $\left\{x: f^{n}(x)=g^{m}(x)\right\}$ is countable, then $f$ and $g$ have a common periodic point. 
Fixed points of contractive type mappings have been studied by a number of authors. B.E. Rhoades [4] has investigated a comparison of different kinds of contractive definitions in the literature. The following theorem is in this direction.

DEFINITION 2.1. Let $X$ be a compact metric space. The function $h: X \times X \rightarrow[0, \infty)$ is said to have property $P_{1}$ if it satisfies the following conditions:

(i): $h(x, y)=0$ if and only if $x=y$,

(ii): if $\lim _{n \rightarrow \infty} x_{n}=x_{0}, \lim _{n \rightarrow \infty} y_{n}=y_{0}$, and $\lim _{n \rightarrow \infty} h\left(x_{n}, y_{n}\right)=0$, then $x_{0}=y_{0}$.

THEOREM 2.2. Let $f$ and $g$ be selfmaps of the unit interval and let $h: I \times I \rightarrow[0, \infty)$ be a function having property $P_{1}$. Suppose $g$ is continuous on $I$ and $A$ is a nonempty closed $g$-invariant subset of $F(f)$. If there exists a real number $a, 0 \leq a<1$ such that for all $x$ and $y$ in $F(f), f$ and $g$ satisfy the following inequality:

$$
\begin{gathered}
h(f x, f y) \leq a \cdot \max \{h(g x, g y), h(g x, f x), h(g y, f y), \\
h(g y, f x), h(f x, g y)\},
\end{gathered}
$$

then $f$ and $g$ have a unique common fixed point.

PROOF. First we show that any two functions $f$ and $g$ satisfying inequality (2.2), have at most one common fixed point. To see this, on the contrary, suppose $q_{1}$ and $q_{2}$ are two different common fixed points of $f$ and $g$. Then using (2.2) we get

$$
\begin{aligned}
h\left(q_{1}, q_{2}\right) & =h\left(f q_{1}, f q_{2}\right) \\
& \leq a \cdot \max \left\{h\left(g q_{1}, g q_{2}\right), h\left(g q_{1}, f q_{1}\right), h\left(g q_{2}, f q_{2}\right), h\left(g q_{2}, f q_{1}\right), h\left(f q_{1}, g q_{2}\right)\right\},
\end{aligned}
$$

implying

$$
\begin{aligned}
h\left(q_{1}, q_{2}\right) & \leq a \cdot \max \left\{h\left(q_{1}, q_{2}\right), h\left(q_{1}, q_{1}\right), h\left(q_{2}, q_{2}\right), h\left(q_{2}, q_{1}\right)\right\} \\
& =a \cdot \max \left\{h\left(q_{1}, q_{2}\right), h\left(q_{2}, q_{1}\right)\right\} .
\end{aligned}
$$

In a similar manner we may show

$$
h\left(q_{2}, q_{1}\right) \leq a \cdot \max \left\{h\left(q_{1}, q_{2}\right), h\left(q_{2}, q_{1}\right)\right\}
$$

hence we shall have

$$
\max \left\{h\left(q_{1}, q_{2}\right), h\left(q_{2}, q_{1}\right)\right\} \leq a \cdot \max \left\{h\left(q_{1}, q_{2}\right), h\left(q_{2}, q_{1}\right)\right\} .
$$

This is impossible unless $\max \left\{h\left(q_{1}, q_{2}\right), h\left(q_{2}, q_{1}\right)\right\}=0$, which implies that $h\left(q_{1}, q_{2}\right)=0$, and hence $q_{1}=q_{2}$, a contradiction. Now we show that $f$ and $g$ have a common fixed point. Without loss of generality we may assume that $A=F(f)$. It is clear that the set $B=\bigcap_{n=1}^{\infty} g^{n}(A)$ is a closed 
nonempty subset of $A$, and that $g^{2}(B)=B$. If for some $y_{0} \in B, f\left(y_{0}\right)=g\left(y_{0}\right)$, then $y_{0}$ is a common fixed point of $f$ and $g$, so we may assume that $f(y) \neq g(y)$ for all $y \in B$. Let $y_{1}$ be an arbitrary point of $B$, and $x_{2} \in B$ be such that $g^{2}\left(x_{2}\right)=y_{1}$. Since $g(y) \neq f(y)$ for all $y \in B$, if we let $x=y_{1}$ and $y_{2}=g\left(x_{2}\right)$, then $g\left(y_{2}\right)=y_{1}=f\left(y_{1}\right)$. Since $h\left(g y_{2}, f y_{2}\right)=h\left(f y_{1}, f y_{2}\right)$, from inequality (2.2) we have

$$
\begin{aligned}
h\left(f y_{1}, f y_{2}\right) \leq & a \cdot \max \left\{h\left(g y_{1}, g y_{2}\right), h\left(g y_{1}, f y_{1}\right), h\left(g y_{2}, f y_{2}\right),\right. \\
& \left.h\left(g y_{2}, f y_{1}\right), h\left(f y_{1}, g y_{2}\right)\right\} \\
& \left.=a \cdot \max \left\{h\left(g y_{1}, g y_{2}\right), h\left(g y_{1}, f y_{1}\right), h\left(g y_{2}, f y_{2}\right), 0\right)\right\} \\
& =a \cdot \max \left\{h\left(g y_{1}, f y_{1}\right), h\left(g y_{2}, f y_{2}\right)\right\},
\end{aligned}
$$

implying $h\left(g y_{2}, f y_{2}\right) \leq a \cdot h\left(g y_{1}, f y_{1}\right)$. If we define $\phi: B \rightarrow[0, \infty)$ by $\phi(x)=h(g x, f x)$, then we have $\phi\left(y_{2}\right) \leq a \cdot \phi\left(y_{1}\right)$. Suppose $x_{3} \in B$ be such that $g^{2}\left(x_{3}\right)=y_{2}$. By taking $x=y_{2}$ and $y_{3}=g\left(x_{3}\right)$, similarly we can show that $\phi\left(y_{3}\right) \leq a \cdot \phi\left(y_{2}\right) \leq a^{2} \cdot \phi\left(y_{1}\right)$. By repeating this process we obtain a bounded sequence $\left\{y_{n}\right\}_{n=1}^{\infty}$ in $B$. Suppose $\left\{y_{n_{k}}\right\}_{k=1}^{\infty}$ is a convergent subsequence of $\left\{y_{n}\right\}_{n=1}^{\infty}$ and $\lim _{k \rightarrow \infty} y_{n_{k}}=y_{0}$. Since $g$ is continuous on $I$,

$$
\begin{aligned}
\lim _{k \rightarrow \infty} h\left(g y_{n_{k}}, y_{n_{k}}\right) & =\lim _{k \rightarrow \infty} h\left(g y_{n_{k}}, f y_{n_{k}}\right) \\
& =\lim _{k \rightarrow \infty} \phi\left(y_{n_{k}}\right) \\
& \leq \lim _{k \rightarrow \infty} a^{n_{k}-1} \cdot \phi\left(y_{1}\right)=0 .
\end{aligned}
$$

So $g\left(y_{0}\right)=y_{0}$, and $y_{0} \in B \subseteq F(f)$, hence $y_{0}$ is a common fixed point of $f$ and $g$.

REMARK 2.1. There is nothing in the proof of Theorem 2.2 that requires the underlying space to be the unit interval. However in our proof the compactness of the unit interval is used. Therefore one can let the underlying space to be a compact metric space. Also condition $P_{1}$ is not a necessary condition and it may be replaced with weaker conditions when $f$ and $g$ are nice. For example if $h, f$ are nice enough that the function $\phi$ defined in Theorem 2.2 attains its minimum at some point $y_{1} \in B$, then we can find a point $y_{2} \in B$ such that $\phi\left(y_{2}\right)<\phi\left(y_{1}\right)$ a contradiction, so $f$ and $g$ must have a common fixed point. If we are not concerned about the uniqueness of the common fixed point, similar to the proof of Theorem 2.2 we have the following.

THEOREM 2.3. Suppose $f$ and $g$ are two selfmaps of a compact metric space $X$ and let $h: X \times X \rightarrow[0, \infty)$ be a function having property $P_{1}$. Suppose also that $g$ is continuous on $X$ and $A$ is a nonempty closed $g$-invariant subset of $F(f)$. If there exists a real number $a, 0 \leq a<1$ such that for all $x$ and $y$ in $A, f$ and $g$ satisfy the following inequality:

$$
\begin{gathered}
h(f x, f y) \leq a \cdot \max \{h(g x, g y), h(g x, f x), h(g y, f y), \\
h(g y, f x), h(f x, g y)\},
\end{gathered}
$$


then $f$ and $g$ have a common fixed point.

The conclusion of Theorem 2.3 is not valid if inequality (2.3) is replaced with

$$
\begin{gathered}
h(f x, f y) \leq \max \{h(g x, g y), h(g x, f x), h(g y, f y), \\
h(g y, f x), h(f x, g y)\} .
\end{gathered}
$$

The following example illustrates this.

EXAMPLE 2.1. Let $f$ and $g$ be defined on the unit interval as follows:

$$
\begin{aligned}
& g(x)= \begin{cases}(8 x+15) / 24 & 0 \leq x \leq 3 / 4 \\
(-32 x+31) / 8 & 3 / 4<x \leq 13 / 16, \\
2 x-1 & 13 / 16<x \leq 7 / 8 \\
3 / 4 & 7 / 8<x \leq 1,\end{cases} \\
& f(x)= \begin{cases}(8 x+39) / 48 & 0 \leq x<3 / 4, \\
3 / 4 & x=3 / 4 \\
(-32 x+39) / 16 & 3 / 4<x \leq 13 / 16, \\
x & 13 / 16<x \leq 7 / 8, \\
7 / 8 & 7 / 8<x \leq 1 .\end{cases}
\end{aligned}
$$

Define $h(x, y): I \times I \rightarrow[0, \infty)$ as $h(x, y)= \begin{cases}0 & \text { if } x=y \\ x+y & \text { if } x \neq y\end{cases}$

One can easily check that $h$ has property $P_{1}, f(x)= \begin{cases}(1+g(x)) / 2 & \text { if } x \neq 3 / 4, \\ 3 / 4 & \text { if } x=3 / 4,\end{cases}$ and $F(f)=\{3 / 4\} \cup[13 / 16,7 / 8]$. Let $A=\{3 / 4,7 / 8\}$, then $A$ is a closed $g$-invariant subset of $F(f)$ and for every $x \neq y$ in $A$ we have

$$
\begin{array}{r}
h(f x, f y)=\max \{h(g x, g y), h(g x, f x), h(g y, f y), \\
h(g y, f x), h(f x, g y)\} \leq 13 / \dot{8} .
\end{array}
$$

It is easy to see that $f$ and $g$ do not have a common fixed point.

THEOREM 2.4. Suppose $f$ and $g$ are two selfmaps of a compact metric space $X$ with $g$ continuous, and let $h: X \times X \rightarrow[0, \infty)$ is a function having property $P_{1}$. If for all $x \neq y$ in $X$, $f$ and $g$ satisfy the following inequality:

$$
\begin{gathered}
h(f x, f y)<\max \{h(g x, g y), h(g x, f x), h(g y, f y), \\
h(g y, f x), h(f x, g y)\},
\end{gathered}
$$

then one of the following holds:

(i) either $f$ and $g$ have a common fixed point, 
(ii) or every nonempty closed $g$-invariant subset of $F(f)$ contains a perfect minimal set $B$ such that the functions $\phi_{1}(x)=h(g x, x)$ and $\phi_{2}(x)=h(x, g x)$, do not attain their minimum or maximum on $B$.

PROOF. From inequality (2.4) it follows that for each $x \in F(f)$ with $x \neq g(x)$, we have $h(g x, x)<h\left(g^{2} x, g x\right)$. This implies that $F(f)$ cannot contain any periodic point of $g$ with period greater than 1. Let $A$ be a nonempty closed $g$-invariant subset of $F(f)$, then it should contain a minimal set $B$. If this minimal set is finite, $f$ and $g$ have a common fixed point. Otherwise, $B$ is a perfect set with $g(B)=B$. Since for each $x \in B, h(g x, x)<h\left(g^{2} x, g x\right)$, the functions $\phi_{1}$ and $\phi_{2}$ cannot attain their maximum or minimum on $B$.

LEMMA 2.1. Suppose $h:[0,1] \times[0,1] \rightarrow[0, \infty)$ is a lower semicontinuous function such that $h(x, y)=0$ if and only if $x=y$. Then $h$ satisfies property $P_{1}$.

PROOF. Suppose $\lim _{n \rightarrow \infty} x_{n}=x_{0}, \lim _{n} \rightarrow \infty y_{n}=y_{0}$, and $\lim _{n \rightarrow \infty} h\left(x_{n}, y_{n}\right)=0$. We need to show that $x_{0}=y_{0}$. On the contrary suppose $x_{0} \neq y_{0}$. Let $\delta=\operatorname{dist}\left\{\left(x_{0}, y_{0}\right),\{(x, x): x \in[0,1]\}\right\}$ , and $B_{1}=\overline{B\left(z_{0}, \frac{\delta}{2}\right)}=B\left[z_{0}, \frac{\delta}{2}\right]$, where $z_{0}=\left(x_{0}, y_{0}\right)$. Let $\epsilon$ be an arbitrary positive number. Then there exists a positive integer $\mathcal{N}$ such that for all $n \geq \mathcal{N},\left(x_{n}, y_{n}\right) \in B_{1}$, and $\left|h\left(x_{n}, y_{n}\right)\right|<\epsilon$. Since $h$ is lower semicontinuous and $B_{1}$ is compact, it attains its minimum at some point $(s, t)$ in $B_{1}$. Hence $|h(s, t)|=\mid$ minimum of $h(x, y)$ on $B_{1}|\leq| h\left(x_{\mathcal{N}}, y_{\mathcal{N}}\right) \mid \leq \epsilon$, implying $h(s, t)=0$. Thus $s=t$, a contradiction.

THEOREM 2.5. Let $f$ and $g$ be commuting selfmaps of $[0,1]$ with $g$ continuous. If $A$ is a nonempty closed $g$-invariant subset of $F(f)$, then one of the following holds:

(i) either $P(f) \cap P(g) \neq \emptyset$,

(ii) or there is a perfect set $A_{1} \subseteq(A \cap R(g) \cap P(f))$.

PROOF. Take $x_{0} \in A$. For every positive integer $n, g^{n}\left(x_{0}\right) \in A$. Thus $O\left(g, x_{0}\right) \subseteq A$ implying $\omega\left(g, x_{0}\right) \subseteq \bar{A}=A$. Clearly $\omega\left(g, x_{0}\right)$ is nonempty and is invariant under $g$. Thus it contains a minimal $g$-invariant subset $A_{1}$. Obviously $A_{1} \subseteq(A \cap R(g))$. If $A_{1}$ is a finite set, then it is the orbit of a periodic point of $g$, implying $\emptyset \neq(P(g) \cap A) \subseteq P(f) \cap P(g)$. Otherwise $A_{1}$ is a perfect set contained in $A \cap R(g)$.

REMARK 2.2. As in the proof of Theorem 2.5, if $f$ and $g$ have no common periodic point then there exits a perfect set $A_{1}$ contained in $A \cap R(g) \cap P(f)$. Since $f$ and $g$ do not have common periodic points and, for a continuous function on a compact interval $\overline{P(f)}=\overline{P(g)}$ ( see Coven and Hedlund [5] ), we have $A_{1} \subseteq A \cap \overline{R(g)}=A \cap \overline{P(g)}=A \cap[\overline{P(g)} \backslash P(g)]$. From this it follows that if $\overline{P(g)} \backslash P(g)$ does not contain a perfect set, then $f$ and $g$ must have a common periodic point. In particular, for two commuting continuous functions if either of $\overline{P(g)} \backslash P(g)$ or $\overline{P(f)} \backslash P(f)$ does not contain a perfect set, then $f$ and $g$ must have a common periodic point.

Consider the following ordering of positive integers: 


\section{$1,2,4,8, \ldots \ldots ., 7 \cdot 8,5 \cdot 8,3 \cdot 8, \ldots, 7 \cdot 4,5 \cdot 4,3 \cdot 4, \ldots, 7 \cdot 2,5 \cdot 2,3 \cdot 2, \ldots, 7,5,3$.}

A.N. Sarkowskii has proven that if $m$ is to the left of $n$ in the above ordering and $f$ has a periodic point of period $n$, then $f$ must have a periodic point of period $m$ (see Stefan [6]). Let $P_{n}(f)=\left\{x \in[0,1]: f^{n}(x)=x\right\}$. The Sarkovskii's theorem immediately implies that if $P_{1}(f)=P_{2}(f)$, then $P_{1}(f)=P_{n}(f)$ for every $n>1$. Suppose $f$ and $g$ are two commuting continuous selfmaps of the unit interval which do not have a common periodic point. We claim that $f$ and $g$ should have periodic points of all even orders. To see this, on the contrary suppose that $n=2^{k} \cdot r(r$ odd, $k \geq 1)$ and $f$ has no periodic point of order $n$. By Sarkowskii's theorem $P_{2 \cdot r}(f)=P_{r}(f)$ implying $P_{1}\left(f^{r}\right)=P_{2}\left(f^{r}\right)$, hence $P_{n}\left(f^{r}\right)=P_{1}\left(f^{r}\right)$ for any $n>1$. Thus we have $P\left(f^{r}\right)=\bigcup_{n=1}^{\infty} P_{n}\left(f^{r}\right)=P_{1}\left(f^{r}\right)$ which implies $\overline{P\left(f^{r}\right)} \backslash P\left(f^{r}\right)=\emptyset$. Since $g$ commutes with $f$, it also commutes with $f^{r}$. Thus $g$ and $f^{r}$ should have a common periodic point which is also a common periodic point of $f$ and $g$. We may interchange the roles of $f$ and $g$. This implies that either $f$ and $g$ have a common periodic point or both have a rich orbit structure. By a rich orbit structure we mean that they have a homoclinic point and positive topological entropy ( see Block [7] ). Thus we have the following:

THEOREM 2.6. Suppose $f$ and $g$ are two commuting continuous selfmaps of the unit interval. Then one of the following holds:

(i) either $P(f) \cap P(g) \neq \emptyset$,

(ii) or both $f$ and $g$ have periodic points of all even orders.

ACKNOWLEDGMENT: I would like to express my sincere thanks to professor B.E. Rhoades for his valuable suggestions in improving the paper, especially on the comments that improved Theorems 2.2 and 2.3. This work also was completed while the author was spending his sabbatical at the University of North Carolina at Wilmington. The hospitality of its Department of Mathematics as well as the financial support of Isfahan University are greatly acknowledged.

AUTHOR'S PRESENT ADDRESS: Mathematical Sciences Department, The University of North Carolina at Wilmington, Wilmington, NC 28403-3297.

\section{REFERENCES}

1. BOYCE, W.M., Commuting functions with no common fixed point, Trans. Amer. Math. Soc. 137(1969), 77-92.

2. HUNEKE, J.P., On common fixed points of commuting functions on an interval, Trans. Amer. Math. Soc. 139(1969), 371-381.

3. SCHWARTZ, A.J., Common periodic points of commuting functions, Michigan Math. J. 12(1965), 353-355. 
4. RHOADES, B.E., A comparison of various definitions of contractive mappings, Trans. Amer. Math. Soc. 226(1977), 257-290.

5. COVEN, E.M., and HEDLUND, G. A., $\bar{P}=\bar{R}$ for maps of the interval, Proc. Amer. Math. Soc. 79(1980), 316-318.

6. STEFAN, P., A theorem of Sarkowskii on the existence of periodic orbits of continuous endomorphisms of the real line, Comm. Math. Phys. 54(1977), 237-248.

7. BLOCK, L., Homoclinic points of mappings of the interval, Proc. Amer. Math. Soc. 72 (1978), 576-580. 


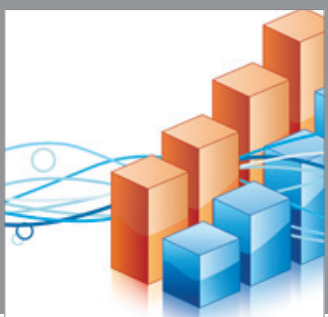

Advances in

Operations Research

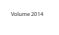

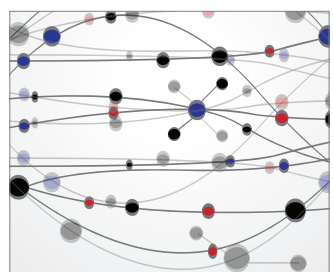

\section{The Scientific} World Journal
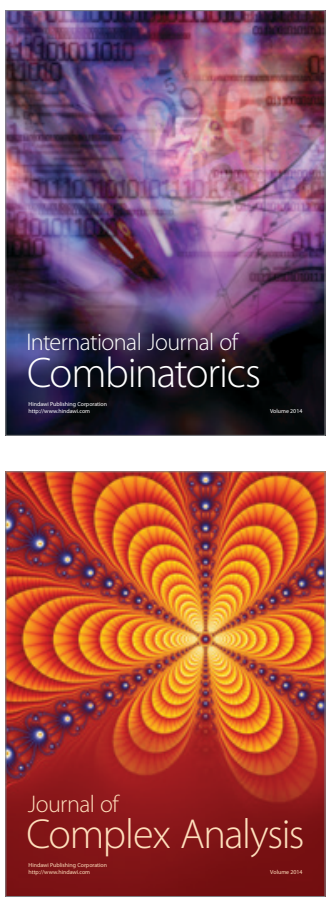

International Journal of

Mathematics and

Mathematical

Sciences
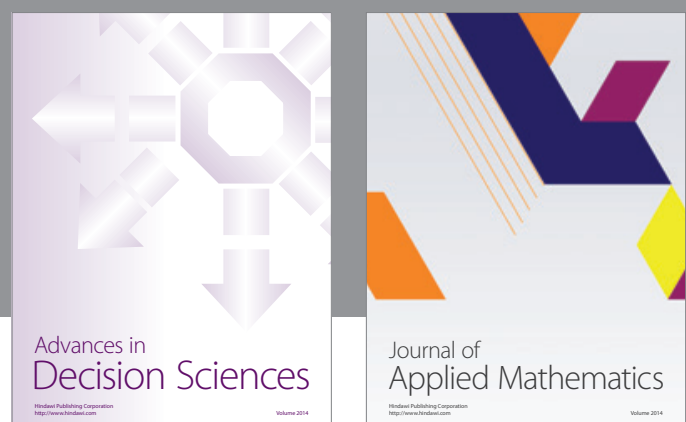

Journal of

Applied Mathematics
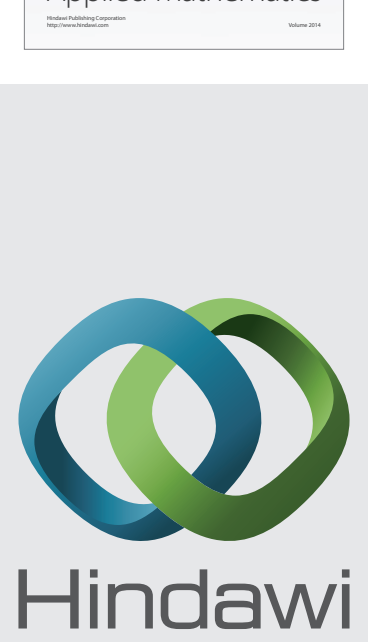

Submit your manuscripts at http://www.hindawi.com
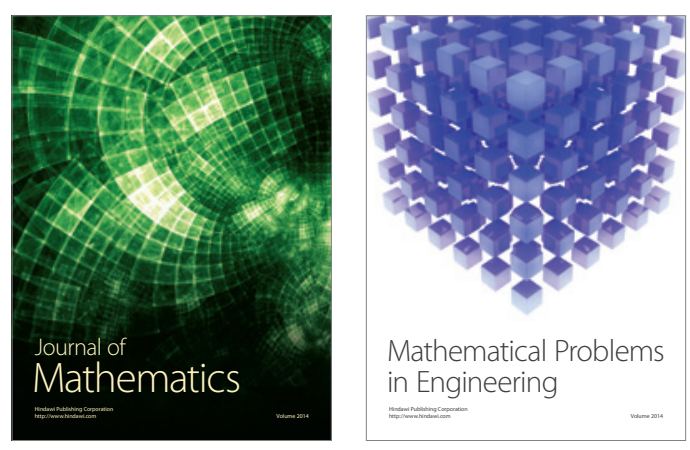

Mathematical Problems in Engineering
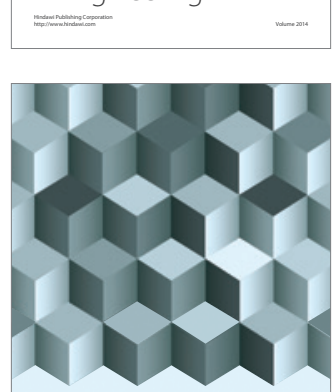

Journal of

Function Spaces
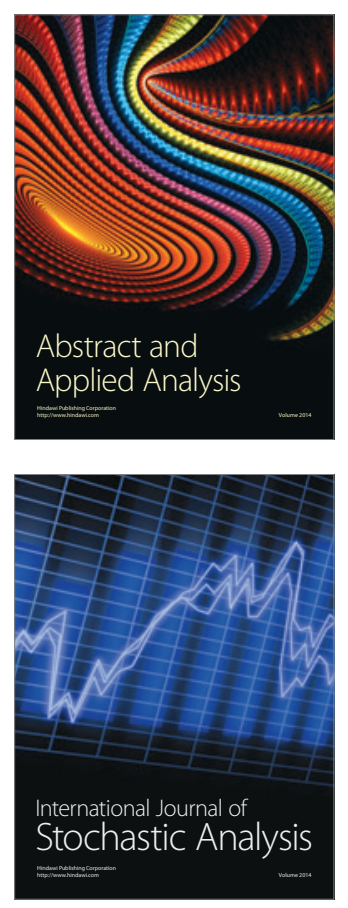

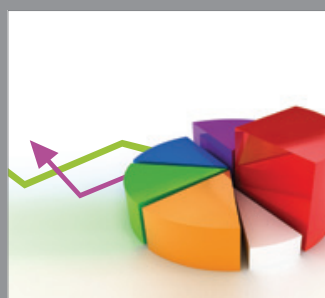

ournal of

Probability and Statistics

Promensencen
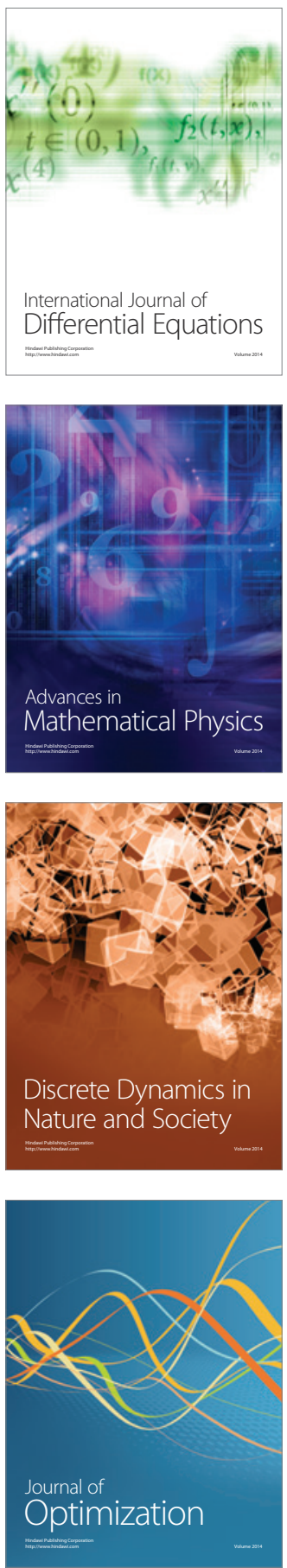